\title{
Bi-Lateral Hand Compartment Syndrome Secondary to Autoimmune Disorder in a Former High School Multi-Sport Athlete
}

Alyssa Vaniman, MS, LAT, ATC

*California State University, Los Angeles, Los Angeles, CA

\begin{abstract}
Patient is a 21-year-old female former multiple-sport athlete who has suffered from the development of multiple cases of compartment syndrome throughout their body. At the time the medical staff encountered the patient, they were diagnosed with bilateral hand compartment syndrome. An autoimmune disorder known as Scleromyositis was diagnosed as the source of the multiple diagnosed cases of compartment syndrome. Prior to surgical intervention, the patient was unable to complete everyday tasks as a student. As pressure within their hypothenar, thenar, and interosseous compartments grew, the patient was no longer able to complete everyday fine motor skill tasks. A post-surgical rehabilitation plan was developed and implemented to improve the patient's dexterity, grip strength, and range of motion. The patient successfully regained strength and dexterity by 8 -week post-surgical intervention. Currently, the patient has not shown any recurrent signs and symptoms of compartment syndrome returning to any anatomical structure. However, it has been roughly four years since their diagnosis of Scleromyositis, they are no longer on immune suppressants, and their primary care physician found no evidence of the autoimmune disorder.
\end{abstract}

Key Phrases

College and University Patient Population, Diagnostic Testing and Physical Examination: Upper Extremity

\section{Correspondence}

Alyssa Vaniman, 11 S. Barranca Ave, West Covina, CA 91791.

E-mail: Alyssa.vaniman@gmail.com.

Twitter: @thatladylyss

\section{Full Citation}

Vaniman A. Bi-lateral hand compartment syndrome secondary to autoimmune disorder in a former high school multi-sport athlete. Clin Pract Athl Train. $2021 ; 4(1)$ : $\quad$ 57-60. https://doi.org/10.31622/2021/0004.1.7.

Submitted: October 27, 2020 Accepted: April 8, 2021.

\section{INTRODUCTION}

According to the National Institutes of Health, approximately 24 million people in the United States suffer from an autoimmune disorder. ${ }^{1}$ In a healthy person, the immune system protects the body against disease and infection. However, in

Copyright $(\subsetneq$ by Indiana State University All rights reserved. ISSN Online 2577-8188 an individual who is diagnosed with an autoimmune disease, the immune system does not function properly, and wrongly starts attacking healthy cells. Scleromyositis is a unique and very rare overlap autoimmune disorder. Scleromyositis is classified as a cross-over disorder because the disease presents with both symptoms of scleroderma and myositis. ${ }^{2}$ Scleroderma is characterized by excessive collagen production and storage within a person's connective tissues. ${ }^{3}$ This excess collagen is coupled with inflammation of the muscles caused by symptoms of myositis. Scleroderma can develop within different anatomical structures throughout the body. ${ }^{3}$ Depending on where the increase in collagen production takes place, this can lead to the inability to compete in athletics or even complete tasks associated with daily life. Due to the excessive amount of collagen production within the connective tissues paired with the significant amount of inflammation of muscle within the hand, the patient developed bilateral compartment syndrome of their hypothenar, thenar, and interosseous compartments. This inflammation and collagen production lead to the patient being unable to hold a pencil, drive, brush their hair, or compete in extramural athletics without severe pain. This particular patient had been managing developing compartment syndrome in multiple parts of their body without a proper diagnosis for years. The lack of answers from medical providers coupled with continued pain took a significant toll on the patient's overall quality of life.

\section{PATIENT INFORMAITON}

Patient is a 21-year old female college senior participating in extramural athletics. The patient was a former high school multi-sport athlete who participated in repetitive impact sports for 
multiple years (soccer, cross-country, basketball). The patient has a previous history of compartment syndrome in their anterior and superficial posterior compartment of the lower limbs as well as in the dorsal and superficial volar compartments of the forearms. Treatment included 12 fasciotomies across the lower and upper extremities of their body in total over five years. The patient also has a previous history of endometriosis in addition to multiple cases of compartment syndrome. The medical team was introduced to the patient in the athletic training clinic after a formal diagnosis of scleromyositis was issued by the patient's primary physician back home. Following this diagnosis, the patient was referred to an immunologist and rheumatologist to rule out the differential diagnosis of carpal tunnel syndrome, median nerve compression at the elbow, ulnar or cubital tunnel syndrome, brachioplexus pathology, or spinal stenosis. The patient stated that proper diagnosis was achieved after a series of extensive blood work to diagnosis the autoimmune disorder and compartment pressure measurement to diagnosis the bilateral compartment syndrome of the thenar, hypothenar, and interosseous compartments. As an out-of-state resident, the patient was making numerous trips to their home state of Wisconsin to get proper testing, followups, and formal visits with their primary physician and care team. The medical team was introduced to the patient after surgery to assist them with post-surgical rehabilitation.

\section{INTERVENTION}

To relieve the pain, diminished sensation, loss of grip strength, and dexterity, the patient underwent three simultaneous fasciotomies of the thenar, hypothenar, and interosseous compartments of both hands. When the patient first reported to the athletic training clinic, to the athletic training clinic, clinic staff (or whomever) conducted a preoperative evaluation. For the presurgical evaluation, the following assessment

Copyright $($ by Indiana State University All rights reserved. ISSN Online 2577-8188 tools/diagnostic tools were used: Sollerman Hand Function test ${ }^{4}$, hand-grip dynamometer, manual muscle testing, goniometry, blood pressure, reflexes, myotomes, and dermatomes, pulse oximeter, heart rate, and hand and forearm girth measurements. Because the patient would undergo anesthesia, medical staff chose to include vitals in pre and postoperative assessment to accurately monitor for adverse reactions following surgery. Two weeks post-surgery, the patient returned to the athletic training clinic for re-evaluation. The patient underwent the same preoperative qualitative and quantitative testing, and SOAP (explained in parenthesis) notes were completed every two weeks to monitor the patient's postoperative results. Medical staff waited to implement the developed protocol until the patient was pain-free or $0 / 10$ on a numeric pain scale, which was predicted to happen threeweeks post-surgery. Due to pain limitations, the developed rehabilitation was not implemented until week four. Bi-weekly assessments and nerve flossing exercises were recommended in the interim. At four weeks postoperative, the patient was pain-free and able to start a rehabilitation regimen. Rehabilitation consisted of nerve flossing (ulnar, median, radial), passive and restive range of motion exercises of the wrist, 6-way wrist movements (flexion, extension, radial/ulnar deviation, supination, pronation), water cup pick up with various amounts of liquid, coloring in an adult coloring book with multiple writing implements, and the use of progressive resistive exercise putty. Formal rehabilitation sessions took place once a week in the athletic training clinic where the majority of these exercises were performed. The patient was encouraged to color and complete nerve flossing multiple times a week.

\section{OUTCOMES}

After surgical intervention, the patient's nerve function fully returned after 5 weeks. The patient stated that their range of motion and strength 
returned to functional levels 8-10 weeks postoperative. However, the patient stopped coming into the athletic training clinic for scheduled rehabilitation appointments after seven weeks due to patient graduating and moving out of state. The patient's relocation also meant testing values and SOAP notes at four weeks and six-week postoperative were unable to be completed. However, medical staff maintained communication with the patient for postoperative follow-up. After the surgical intervention, the patient has not redeveloped any signs or symptoms of compartment syndrome in the hands. As of June 2020, patient's previously diagnosed autoimmune disorder is asymptomatic without the use of immunosuppressant medication. The patient was closely monitored by their primary care physician upon returning home and continues to have no symptoms of compartment syndrome.

\section{DISCUSSION}

The diagnosis of the injury in this patient's case was extremely complex. Without a direct mechanism of injury and conflicting symptoms, the diagnosis remained unclear until the rheumatologist and immunologist confirmed with differential diagnostic testing. The patient and medical team also faced additional challenges during treatment: the patient's status as an outof-state resident, lack of insurance coverage due to patient's out-of-state status, and a lack of expertise in autoimmune disorders at the treatment facility. This patient's case was unique with regard to epidemiology - the case presentation was individual and atypical. Due to the complex nature of this case, medical professionals from various specialties were involved in the diagnosis, treatment, and rehabilitation of this patient. It is important to note that an accurate diagnosis would have been unlikely without proper referrals and the patient's quality of life and functional capabilities would have continued to suffer. More importantly, without the proper referrals during the diagnosis,

Copyright $\left({ }^{\circ}\right.$ by Indiana State University All rights reserved. ISSN Online 2577-8188 treatment, and rehabilitation phases the patient's quality of life and functional capabilities would have continued to suffer.

\section{CLINICAL BOTTOM LINE}

When diagnosing a patient, referral for clinical lab testing, alternate diagnostic tools, and referral to the appropriate medical professional team could be the defining factors in proper diagnosis and treatment for your patient. Athletic trainers are a valuable piece of the sports medicine team that work collaboratively with other medical professionals in diagnosis and treatment of illness and injury. This case serves as a great reminder that athletic trainers are a critical component of the healthcare team and often serve as facilitators for communication and collaboration among team members, ensuring the patient receives the highest possible level of care.

\section{REFERENCES}

1. The Autoimmune Diseases Coordinating Committee. U.S. Department of Health and Human Services; 2005.

https://www.niaid.nih.gov/sites/default/ files/adccfinal.pdf. Accessed July 7, 2020.

2. Venables PJ. Polymyositis-associated overlap syndromes. Br J Rheumatol. 1996;35(4):305-306. https://doi.org/10.1093/rheumatology 35.4.305.

3. Bhansing $\mathrm{KJ}$, Lammens $M$, Knaapen $H K$, van Riel PL, van Engelen BG, Vonk MC. Scleroderma-polymyositis overlap syndrome versus idiopathic polymyositis and systemic sclerosis: A descriptive study on clinical features and myopathology. Arthritis Res Ther. 2014;16(3):R 111. https://doi.org/10.1186/ar4562. 
4. Sollerman C, Ejeskär A. Sollerman. Hand function test: A standardised method and its use in tetraplegic patients. Scand J

Plast Reconstr Surg Hand Surg.

$2009 ; 1995 ; 29: 167-176$.

https://doi.org/10.3109/0284431950

9034334. 